\title{
Upsurging Paratyphoid Fever: A Study from Southern India
}

\author{
Chaitra Govardhan ${ }^{1}$, Christi Dominic Savio ${ }^{1}$, Susan Verghese ${ }^{2}$ \\ ${ }^{1}$ Department of Pediatrics, Church of South India Hospital, Bengaluru, Karnataka, India \\ ${ }^{2}$ Department of Microbiology, Church of South India Hospital, Bengaluru, Karnataka, India
}

\begin{abstract}
Objectives: Enteric fever is still a major public health problem in an endemic country like India. Studies project that Salmonella Typhi is the most common cause in the pediatric population but with a recent rising trend in the number of cases due to Salmonella Paratyphi A with increased morbidity and mortality.
\end{abstract}

Methods: A retrospective analysis of children who had been infected with laboratory-confirmed Salmonella serotypes of Typhi or Paratyphi A collected from November 2016 to October 2018, isolates underwent serotype confirmation, antimicrobial susceptibility testing.

Results: A total of 29 isolates of Salmonella species were studied, which revealed an emergence of Salmonella Paratyphi A as predominant serotype accounting for $55.2 \%$ (16) cases with remaining $44.8 \%$ (13) cases being Salmonella Typhi.

Conclusions: Physicians should be aware of the increasing incidence of infection due to Salmonella Paratyphi A and treatment options given its widespread antimicrobial resistance. A paratyphoid fever vaccine is urgently needed. Continued surveillance for paratyphoid fever will help guide future prevention and treatment recommendations. $J$ Microbiol Infect Dis 2020; 10(1):18-23.

Keywords: Salmonella Paratyphi A, Enteric fever, Drug resistance, Rising trend

\section{INTRODUCTION}

Salmonella Paratyphi A is the second most common cause of enteric fever after Salmonella Typhi with infection rate between Salmonella Typhi to Salmonella Paratyphi being approximately $5: 1$ [1]. It is estimated that more than 26.9 million enteric fever cases out of which 5.4 million cases caused by paratyphoid occur each year [1]. Previously paratyphoid fever was considered to occur less frequently and to be a more benign disease than compared to typhoid fever. But lately, since 1996, the number of isolations of Salmonella Paratyphi A causing enteric fever cases are showing an increasing trend which has been noticed in India [2]. Recent studies have time and again established that illnesses caused by Salmonella Paratyphi A and Salmonella Typhi are clinically indistinguishable $[3,4]$. Salmonella Paratyphi A infection even though said to present as a milder illness, causes systemic complications in 10\%$15 \%$ of cases, such as meningitis, endocarditis, hepatic abscess, osteomyelitis, and psoas abscess, gallbladder cancer, and pancytopenia [5]. Blood culture always stands as the gold standard for diagnosis and also gives information regarding the antibiotic sensitivity pattern of the isolates. But certain factors such as the cost of the culture, lack of standard culture methods and high incidence of prior administration of antibiotics are impediments in this diagnostic approach in a resource-poor country like India. The most commonly used test in Indian set up is the Widal test, but has very variable sensitivity and specificity and therefore presents a diagnostic problem in interpretation. Salmonella Paratyphi A with nalidixic acid, ceftriaxone, and multidrug resistance has also been recently reported in India. Among

Correspondence: Dr. Chaitra Govardhan, Department of Pediatrics, Church of South India Hospital, Bengaluru, Karnataka, India. Address: Post Bag No.4, Hazarath Kambhal Posh Road, Bangalore-560051, Karnataka, India 
Salmonella Paratyphi A isolates, nalidixic acid resistance is increasing and also has become predominant in areas of endemicity [6]. Unlike typhoid fever, no vaccine is currently available in the market that provides specific protection against paratyphoid fever. In the absence of effective paratyphoid fever surveillance, clinicians are unable to base treatment decisions on incidence and resistance patterns. The present study was thus undertaken to compare and estimate the current burden, evaluate the clinical profile and changing susceptibility pattern to antimicrobials of Salmonella Typhi and Paratyphi $A$ isolates over a period of 2 years in a tertiary care hospital in a southern part of India.

\section{METHODS}

A retrospective study was carried out at Department of Pediatrics, Church of South India Hospital located in the urban part of Bangalore in Karnataka state. The study included a period of 2 years, from November 2016 to October 2018. Detailed analyses of these 29 culture positive enteric fever cases records were done. Cases that were included in study were those whose were diagnosed based on clinical features followed by isolation of Salmonella Typhi or Salmonella Paratyphi A from blood using BactT/Alert automated blood culture system. Antimicrobial susceptibility testing was done using the Kirby-Bauer disc diffusion method, according to standard guidelines. The mode of clinical presentation, laboratory diagnosis, and antibiotic susceptibility patterns were recorded from the medical records section. Statistical software SPSS 18.0 was used for the analysis of the data. Chi-square for the observed trend was calculated, and the p-value was determined.

\section{RESULTS}

Totally 29 enteric fever cases were analyzed, among which 8 (27.6\%) and 21 (72.4\%) were isolated during each year respectively. In our study, Salmonella Paratyphi A was more frequent serotype isolated accounting for 16 $(55.2 \%)$ of the cases with the remaining 13 (44.8\%) being Salmonella Typhi. The isolation trend changed from Salmonella Typhi to Salmonella Paratyphi A during the study period $(p=0.010$; statistically significant). A drastic upsurge of Salmonella Paratyphi A as the predominant serotype was noticed during the second half of the study period which was carried out for a total period of two years, the proportion of Salmonella Paratyphi A among the total enteric fever cases increased from 1 (6.3\%) case to $15(93.8 \%)$ cases which was statistically significant.

The socio-demographic characteristics are depicted in Table 1. Mean age of presentation was around $8.28+/-4.34$ years with a male to female ratio of 2:1 observed with both serovars. Clustering of cases for both serovars was observed maximum between the months of April to July accounting for almost $54 \%$ of the cases. (Figure 1)

Fever was the most common symptom present in all cases $(100.0 \%)$ of both serovars, followed by vomiting (53.8\%) and loose stools (46.2\%), pain abdomen and myalgia (38.5\%), headache (30.8\%), dry cough (23.1\%) in Salmonella Typhi cases. S. Paratyphi A cases presented with pain abdomen (43.8\%) as a second most common symptom after fever (100\%), followed by cough $(18.8 \%)$ and headache (12.5\%). Hepatosplenomegaly was found in $37.5 \%$ cases and splenomegaly in $31.3 \%$ of $S$. Paratyphi $A$ cases (Table 2).

Investigation findings are depicted in Table 3. Anemia $(50 \%)$ followed by thrombocytopenia $(25 \%)$ and leucopenia $(25 \%)$ were observed in Salmonella Paratyphi A cases. Leucopenia $(50 \%)$ followed by anemia $(46.2 \%)$ and thrombocytopenia (38.5\%) with 2 cases of Dengue co-infection proved by IgM ELISA were observed in Salmonella Typhi cases. The antimicrobial sensitivity of both serovars has been depicted in Table 4. It is interesting to note that all the serovars were $100 \%$ sensitive to ceftriaxone, chloramphenicol, and cotrimoxazole. Salmonella Paratyphi A showed $100 \%$ resistance to nalidixic acid suggesting low sensitivity to fluoroquinolones ( $p$ value $=0.078$ ).

\section{DISCUSSION}

India is a vast country with considerable geographic, socio-economic and religious diversity. However, enteric fever still remains endemic throughout the country and places a challenge on healthcare facilities to curtail this problem. Humans are the only predominant host for typhoidal salmonellas, transmitted through contaminated food or water with human feces. 
Children with typhoidal illness generally present at a later stage of the disease and combined with the lack of standard diagnostic facilities, relying on suboptimal tests and widespread use of irrational antibiotics in the community without prescription are major issues concerning the appropriate management and its outcome. In India, enteric fever is more prevalent in urban areas than compared to rural setup, with an incidence approaching almost one percent of the population annually in some endemic areas of the country [7]. Earlier Salmonella enterica serovar Paratyphi $A$ was a less frequent cause of enteric fever, with isolation rates being around $3-17 \%$ from India. But few recent studies have revealed a rising incidence in the enteric fever cases due to Salmonella Paratyphi A since the year 1996 [8]. The varied reasons for the emergence of paratyphoid fever in India are however unknown and cannot be readily explained by either because of improved diagnostic methods, or better vaccination coverage, or the effect of improved water and sanitation measures. The emergence of nalidixic acid-resistant Salmonella Paratyphi A is not surprising, given the easy availability of fluoroquinolones as an over the counter drug throughout the country. In the absence of an effective vaccine against Salmonella Paratyphi $A$, prevention of the same is extremely challenging.

Clinically, the paratyphoid fever has three stages: (A) An early stage marked by high grade fever; (B) A toxic second stage with abdominal pain and intestinal symptoms, and (C) A long period of gradual defervescence. Among this, the toxic stage is the most important as there are an $1-10 \%$ chances of intestinal perforation, hemorrhage or inflammatory destruction [9]. The infection may develop cardiac complications and sometimes even fatal in children. General symptoms of Salmonella Paratyphi A infection include fever, headache, diarrhea or constipation, malaise, anorexia, nausea, dry cough, abdominal pain, chills, raised spots or rashes on the body $[10,11]$. Early identification of the specific causative serovar and updated knowledge of local antimicrobial resistance patterns would be invaluable in guiding rational treatment decisions [12].
This is one of the studies, to our knowledge, attempted to study the epidemiology of paratyphoid fever with that of typhoid fever. It confirms that paratyphoid fever is equally severe illness clinically and most often is caused by nalidixic acid-resistant Salmonella Paratyphi A.

A few retrospective cohort studies have been conducted in India to monitor the similar trends of Salmonella Paratyphi A infection. An increase in isolation of Salmonella Paratyphi A was reported with $11.1 \%$ (2001) to 59\% (2003) from Calicut [13]. Between 2001 to 2003 an unusually high occurrence of Salmonella Paratyphi A infection was reported from Nagpur [13].

A recent study from Chandigarh also showed a similar increasing trend of isolation of Salmonella Paratyphi from January 2003 to April 2007 [14]. Another study conducted in the year 2004 revealed that isolation of Salmonella Paratyphi A (170 cases) was relatively higher than Salmonella Typhi (79 cases) at the Manipal Hospital in Bangalore [15].

Drug resistance in typhoid fever is considered one of the most important factors in determining the morbidity and mortality of the disease. The quinolone group of drugs emerged as the alternative useful drugs for the treatment of multiple drug resistant cases of Salmonella Typhi but unfortunately, ciprofloxacin resistance in the Salmonella Paratyphi A isolates has been reported from various parts of India [16-18]. Adverse impact of Nalidixic acid resistant Salmonella Typhi (NARST) strains, with longer duration of fever, higher frequency of hepatomegaly and elevated liver enzymes with increased Minimum Inhibitory Concentration (MIC) of ciprofloxacin have been reported [19].

All isolates of Salmonella Paratyphi A in our study showed decreased susceptibility to Ciprofloxacin that may be associated with delayed defervescence, persistence of positive fecal culture results even after antimicrobial therapy, clinical relapse, and the need for additional treatment. The global emergence of multidrug-resistant strains with decreased susceptibility to fluoroquinolones with poor clinical response despite disk sensitivity was reported by Bhan MK et al in 2005 [7]. 
Table 1. Correlation of year, age and Gender distribution in relation to causative serovar of the pediatric population studied.

\begin{tabular}{lcccc}
\hline Variables & S. Typhi $(\mathrm{n}=13)$ & S. Paratyphi A $(\mathrm{n}=16)$ & Total $(\mathrm{n}=29)$ & P value $^{*}$ \\
\hline 2016 November-2017 October & $7(53.8 \%)$ & $1(6.3 \%)$ & $8(27.6 \%)$ & $0.010^{*}$ \\
2017 November -2018 October & $6(46.2 \%)$ & $15(93.8 \%)$ & $21(72.4 \%)$ & \\
Age (years) & & & & \\
\multicolumn{1}{c}{$1-10$} & $9(69.2 \%)$ & $12(75 \%)$ & $21(72.4 \%)$ & 1.000 \\
\multicolumn{1}{c}{$11-20$} & $4(30.8 \%)$ & $4(25 \%)$ & $8(27.6 \%)$ & \\
Female & $4(30.8 \%)$ & $5(31.3 \%)$ & $9(31 \%)$ & 1.000 \\
Male & $9(69.2 \%)$ & $11(68.8 \%)$ & $20(69 \%)$ & \\
\hline${ }^{*}$ Chi-Square/Fisher Exact Test & & &
\end{tabular}

Table 2. Distribution of clinical features in relation to causative serovar of the pediatric population studied.

\begin{tabular}{|c|c|c|c|c|}
\hline Clinical features & S. Typhi $(\mathrm{n}=13)$ & S. Paratyphi A $(\mathrm{n}=16)$ & Total $(n=29)$ & $\mathrm{P}$ value ${ }^{*}$ \\
\hline Fever & $13(100 \%)$ & $16(100 \%)$ & $29(100 \%)$ & 1.000 \\
\hline Pain abdomen & $5(38.5 \%)$ & $7(43.8 \%)$ & 12 (41.4\%) & 0.774 \\
\hline Vomiting & $7(53.8 \%)$ & $4(25 \%)$ & $11(37.9 \%)$ & 0.111 \\
\hline Diarrhea & $6(46.2 \%)$ & $0(0 \%)$ & $6(20.7 \%)$ & $0.004^{* *}$ \\
\hline Myalgia & $5(38.5 \%)$ & $0(0 \%)$ & $5(17.2 \%)$ & $0.011^{*}$ \\
\hline Headache & $4(30.8 \%)$ & $2(12.5 \%)$ & $6(20.7 \%)$ & 0.364 \\
\hline Cough & $3(23.1 \%)$ & $3(18.8 \%)$ & $6(20.7 \%)$ & 1.000 \\
\hline Splenomegaly & $4(30.8 \%)$ & $5(31.3 \%)$ & $9(31 \%)$ & \multirow{4}{*}{0.833} \\
\hline Hepatomegaly & $4(30.8 \%)$ & $4(25 \%)$ & $8(27.6 \%)$ & \\
\hline Absent & $2(15.4 \%)$ & $1(6.3 \%)$ & $3(10.3 \%)$ & \\
\hline Both & $3(23.1 \%)$ & $6(37.5 \%)$ & $9(31 \%)$ & \\
\hline
\end{tabular}

${ }^{*}$ Chi-Square/Fisher Exact Test

Table 3. Investigation profile in study population infected with Salmonella serovars.

\begin{tabular}{|c|c|c|c|c|}
\hline Variable & S. Typhi, $(n=13)$ & S. Paratyphi A, $(n=16)$ & Total $(n=29)$ & $P$ value* \\
\hline Anemia & $6(46.2 \%)$ & $8(50 \%)$ & $14(48.3 \%)$ & 0.837 \\
\hline Leucopenia & $7(53.8 \%)$ & $4(25 \%)$ & $11(37.9 \%)$ & 0.143 \\
\hline Thrombocytopenia & $5(38.5 \%)$ & $4(25 \%)$ & $9(31 \%)$ & 0.688 \\
\hline Dengue & $2(15.4 \%)$ & $0(0 \%)$ & $2(6.9 \%)$ & 0.192 \\
\hline Coinfection & $2(15.4 \%)$ & $0(0 \%)$ & $2(6.9 \%)$ & 0.192 \\
\hline
\end{tabular}

${ }^{*}$ Chi-Square/Fisher Exact Test 
Table 4. Antimicrobial sensitivity pattern of Salmonella serovars.

\begin{tabular}{|c|c|c|c|c|}
\hline Sensitivity & S. Typhi & S. Paratyphi A & Total & $P$ value* \\
\hline \multicolumn{5}{|l|}{ Cephalosporins } \\
\hline Cotrimoxazole & $13(100 \%)$ & $16(100 \%)$ & $29(100 \%)$ & 1.000 \\
\hline \multicolumn{5}{|l|}{ Chloramphenicol } \\
\hline Nalidixic acid & $3(23.1 \%)$ & $0(0 \%)$ & $3(10.3 \%)$ & 0.078 \\
\hline Ciprofloxacin (low sensitivity) & $10(77.0 \%)$ & $16(100 \%)$ & $26(89.7 \%)$ & 0.078 \\
\hline
\end{tabular}

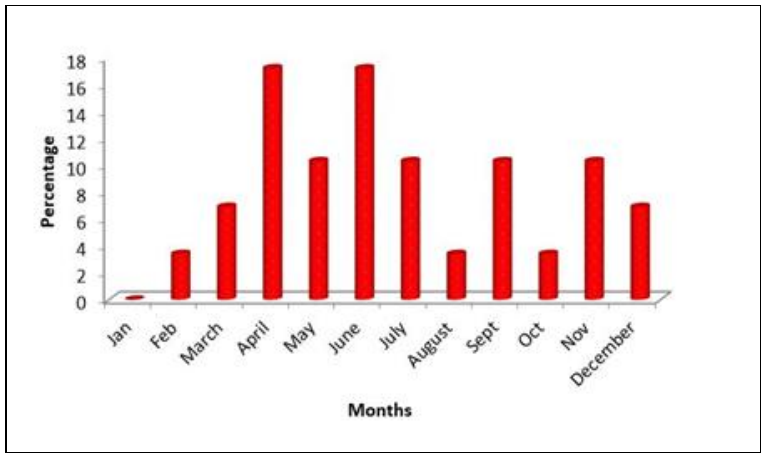

Figure 1. Bar chart depicting the annual distribution of Salmonella serovars.

For the prevention of paratyphoid fever, outreach efforts into the peripheries and effective health messages to the community to be spread throughout the country are essential. Effective vaccines against both Salmonella Typhi as well as Paratyphi A are urgently needed. Consideration should be given to including paratyphoid fever as a specific nationally notifiable disease, in order to take up more stringent actions to prevent further outbreaks. National level laboratory-based epidemiologic surveillance for paratyphoid fever, such as is currently performed for typhoid fever, is essential to monitor trends, resistance pattern and also to develop future prevention and treatment strategies.

\section{Conclusion}

In conclusion, though paratyphoid fever was previously considered to be a relatively milder as compared to typhoid fever, our findings highlight that both had a similar presentation, and also stresses the importance of changing trends in susceptibility pattern of isolates. Since currently, there is no effective vaccination against Salmonella Paratyphi A we also reinforce the importance of various health care systems to implement alternative public health interventions required in educating the general population about preventive measures such as safe drinking water, food, personal hygiene, and sanitation. Antibiotics should be cautiously used according to local susceptibility pattern and indiscriminate use should be discouraged.

\section{ACKNOWLEDGMENTS}

Competing interests: The authors declare no competing interest.

Funding: The authors received no funding for this study.

\section{REFERENCES}

1. Kliegman R, Stanton B, St. Geme J, Schor N, Behrman R, Nelson W. Nelson Textbook of Pediatrics. Philadelphia: Elsevier; 2016; 198:13881393.

2. Chandel D. Drug-Resistant Salmonella enterica Serotype Paratyphi A in India. Emerg Infect Dis 2000; 6(4):420-421.

3. Maskey A, Day J, Tuan P, et al. Salmonella enterica Serovar Paratyphi A and S. enterica Serovar Typhi Cause Indistinguishable Clinical Syndromes in Kathmandu, Nepal. Clin Infect Dis 2006; 42(9):1247-1253.

4. Chowta N, Chowta M. Study of Clinical Profile and Antibiotic Response in Typhoid Fever. Indian Journal of Medical Microbiology 2005; 23(2):125.

5. Bekur R, Vandana K, Shivashankara K, Valsalan R, Sathyanarayanan V. Paratyphoid feverEmerging problem in South India. Asian Pacific J Trop Med 2010; 3(10):815-817.

6. Bhattacharya SS, Dash U. Sudden rise in the occurrence of Salmonella Paratyphi infection in Rourkela, Orissa. Indian J Med Microbiol 2007; 25(1): 78-79. 
7. Bhan M, Bahl R, Bhatnagar S. Typhoid and paratyphoid fever. The Lancet 2005; 366 (9487):749-762.

8. Threlfall E, Fisher I, Berghold C, et al. Trends in antimicrobial drug resistance in Salmonella enterica serotypes Typhi and Paratyphi $A$ isolated in Europe, 1999-2001. Intern J Antimicrob Agent 2003; 22(5):487-491.

9. Vollaard A, Ali S, Widjaja S, et al. Identification of typhoid fever and paratyphoid fever cases at presentation in outpatient clinics in Jakarta, Indonesia. Trans R Soc Trop Med Hyg 2005; 99(6):440-450.

10. Chaudhry R, Chandel D, Pandey A, Dhawan B, Gulati V, Sood R. Utility of PCR in diagnosing complicated cases of unusual clinical manifestations of Salmonella enterica var. paratyphi A. Amer J Med 2005; 118(7):799-800.

11. Walia M, Gaind R, Paul P, Mehta R, Aggarwal P, Kalaivani $M$. Age-related clinical and microbiological characteristics of enteric fever in India. Trans R Soc Trop Med Hyg 2006; 100(10):942-948.

12. Parry C, Hien T, Dougan G, White N, Farrar J. Typhoid Fever. N Eng J Med 2002; 347(22):17701782.

13. Tankhiwale SS, Agrawal G, Jalgaonkar SV. An unusually high occurrence of Salmonella enterica serotype Paratyphi $A$ in patients with enteric fever. Ind J Med Res 2003; 117: 10-12.

14. Joshi S, Amarnath S. Fluoroquinolone resistance in Salmonella Typhi and $S$. Paratyphi A in Bangalore, India. Trans R Soc Trop Med Hyg 2007; 101(3):308-310.

15. Joshi S, Wattal C, Sharma A, Oberoi JK, Prasad KJ. Quinolones drug of choice for enteric fever? Indian Journal of Medical Microbiology. 2004; 22(4): 271-2.

16. Madhulika U, Harish BN, Parija SC. Current pattern in antimicrobial susceptibility of Salmonella Typhi isolates in Pondicherry. Ind $\mathrm{J}$ Med Research. 2004; 120(8): 111-114.

17. Thaver D, Zaidi AK, Critchley J, Madni SA, Bhutta ZA. Fluoroquinolones for treating typhoid and paratyphoid fever (enteric fever). Cochrane Database Systematic Reviews 2005; 2.

18. Kadhiravan T, Wig N, Kapil A, Kabra S, Renuka K, Misra A. Clinical outcomes in typhoid fever: adverse impact of infection with nalidixic acidresistant Salmonella Typhi. BMC Infect Dis 2005;5(1).

19. Thisyakorn U, Mansuwan P, Taylor DN. Typhoid and paratyphoid fever in 192 hospitalized children in Thailand. Am J Dis Child 1987; 141: 862-5. 